\title{
Utility of Neutrophil to Lymphocyte Ratio and Platelet to Lymphocyte Ratio as an Early Predictor of Severe Acute Biliary Pancreatitis
}

\author{
Surendra Shahl, Sanjaya Paudyal ${ }^{1}$, Shanta Bir Maharjan ${ }^{1}$, Shailendra Shah ${ }^{1}$, \\ Jay Narayan Shah ${ }^{1}$ \\ ${ }^{1}$ Department of Surgery, Patan Hospital, Patan Academy of Health Sciences, Kathmandu, Nepal
}

\section{ABSTRACT}

Introduction: Acute severe pancreatitis is associated with increased mortality. Several scoring systems have been used to predict severe acute pancreatitis which are either time-consuming or calculated 48 hours after admission. This study was aimed to assess the utility of neutrophil to lymphocyte ratio and platelet to lymphocyte ratio as an early predictor of severe acute biliary pancreatitis.

Materials and Methods: This was a retrospective cohort study conducted from January 2017 to January 2020. Patients with non-biliary pancreatitis, referred after initial treatment, missed data, and acute pancreatitis with acute cholecystitis or cholangitis were excluded from the study. Data were collected from case sheets. Patients were divided into two groups according to the development of severe acute biliary pancreatitis based on the revised Atlanta Classification. Association of neutrophil to lymphocyte ratio and platelet to lymphocyte ratio with severe acute biliary pancreatitis was assessed using Mann Whitney U-test. $p$-value $<0.05$ was considered as statistically significant.

Results: Total 73 cases included in the study (males/females $=0.55$ ). Sixty-six patients (90.4\%) had mild/moderate acute biliary pancreatitis, and $7(9.6 \%)$ patients had severe acute biliary pancreatitis. There was a development of complications in $7(9.6 \%)$ patients who had severe acute biliary pancreatitis including one mortality. The mean neutrophil to lymphocyte ratio and mean platelet to lymphocyte ratio were high in the severe acute biliary pancreatitis group compared to the nonsevere acute biliary pancreatitis group, however, these differences were not statistically significant.

Conclusions: Neutrophil to lymphocyte ratio and platelet to lymphocyte ratio cannot predict severe acute biliary pancreatitis.

Keywords: Acute biliary pancreatitis; Organ failure; Neutrophil to lymphocyte ratio; Platelet to lymphocyte ratio

\section{Correspondence:}

Dr. Surendra Shah, MS, Mch

Department of Surgery, Patan Hospital,

Patan Academy of Health Sciences, Lagankhel,

Lalitpur.

ORCID ID: 0000-0002-0510-3952

Email: drsurendrashah@gmail.com

Submitted: $14^{\text {th }}$ May 2021

Accepted: $20^{\text {th }}$ June 2021

Source of Support: None

Conflict of Interest: None

Citation: Shah S, Paudyal S, Maharjan SB, Shah S, Shah JN. Utility of Neutrophil to Lymphocyte ratio and platelet to lymphocyte ratio as an early predictor of severe acute biliary pancreatitis. NMJ 2021;4(1):410 3. DOI $10.3126 /$ nmj.v4i1.37094

\section{INTRODUCTION}

Acute pancreatitis (AP) is one of the major causes of gastrointestinal emergencies and about $10 \%$ among them develop severe acute pancreatitis (SAP). The overall mortality of AP is $2.5-7.5 \%$ but increases to 30 to $45 \%$ in SAP. ${ }^{1-3}$ There are many scoring systems to predict SAP. Acute Physiology and Chronic Health Evaluation II (APACHE II) used in intensive care unit which is time-consuming, Ranson score which requires 48 hours and multiple laboratory parameters, and Bedside Index for 
Severity in Acute Pancreatitis (BISAP) which is mainly used to predict mortality in $\mathrm{AP}^{4}$

Complete blood count (CBC) is a routine test for emergency patients available even in the resource-limited center. Recent studies show NLR (neutrophil to lymphocyte ratio) and PLR (platelet to lymphocyte ratio) can predict severe acute biliary pancreatitis. ${ }^{5}$ Local data (from Nepal) on a similar study was not available (on PubMed, Google Scholar, and NepJOL search). Early prediction of SAP helps to guide the clinician in further management and counseling about AP. This study aims to analyze the role of NLR and PLR to predict severe acute biliary pancreatitis (SABP) patients, admitted to Patan Hospital.

\section{MATERIALS AND METHODS}

This was a retrospective cohort study conducted from January 2017 to January 2020 in the Department of Surgery, Patan Hospital affiliated with Patan Academy of Health Sciences, Kathmandu, Nepal. Approval for the study was taken from the Institutional Review Committee of Patan Academy of Health Sciences. The objective of the study was to analyze the predictive role of NLR and PLR in predicting severe acute biliary pancreatitis (ABP) at the time of admission. Patients admitted with the diagnosis of acute biliary pancreatitis in the Department of Surgery during the study period were included in the study. Patients with nonbiliary pancreatitis, history of treatment at another center for the same condition before visiting Patan Hospital, missed data in the file, and acute pancreatitis associated with acute cholecystitis or cholangitis were excluded from the study. All the data were collected from the patient's record file which was retrieved from the medical record department using ICD-K85.1 code (ICD code of acute biliary pancreatitis). Serum amylase and/or lipase, absolute neutrophil count, absolute lymphocyte count, and platelet count were recorded at the time of admission. Radiological (ultrasound and/or computed tomography) findings during hospitalization were recorded. Arterial blood gas, serum creatinine, and blood pressure were recorded at the time of admission and 48 hours after admission.

Acute pancreatitis was defined as the presence of two of the following three: abdominal pain consistent with acute pancreatitis (acute onset of a persistent, severe, epigastric pain often radiating to the back), serum lipase or amylase activity at least three times greater than the upper limit of normal, and characteristic findings of acute pancreatitis on contrast-enhanced computed tomography (CECT) and less commonly magnetic resonance imaging (MRI) or transabdominal ultrasonography. ${ }^{6}$ Acute biliary pancreatitis (ABP) was defined as acute pancreatitis associated with gallstone or gallbladder sludge in the imaging. Organ failure was defined as "Modified Marshall Score" of 2 or $>2$, that is, $\mathrm{PaO}_{2} / \mathrm{FiO}_{2} \leq 300$ (respiratory failure), systolic blood pressure $<90 \mathrm{mmHg}$ which is not responding to intravenous fluid (cardiac failure), and serum creatinine $>1.8 \mathrm{mg} / \mathrm{dl}$ (renal failure) ${ }^{6}$ Severe acute pancreatitis was defined as persistent single or multiple organ failure (among respiratory, cardiovascular, and renal systems) for $>48$ hours of admission. $^{6}$

Patients were divided into two groups according to the development of acute severe pancreatitis. Patients with acute severe pancreatitis were included in Group A and patients with acute moderately severe and mild pancreatitis were included in Group B. Association of NLR and PLR (calculated at the time of admission) with acute severe pancreatitis was assessed using Mann Whitney U-test. The $p$-value $<0.05$ was considered statistically significant.

\section{RESULTS}

There were 88 admissions with the diagnosis of acute biliary pancreatitis during the study period. Among 88, 15 were excluded from the study. Among the excluded cases, acute biliary pancreatitis was associated with acute cholecystitis in 9 patients, missed reports in 3 case sheets, history of treatment at another center for the same illness in 2 patients, and associated with acute cholangitis in one patient. The remaining 73 patients were included in the study for analysis. There were $26(35.6 \%)$ male, and $47(64.4 \%)$ female patients in the study (Table 1$)$.

Table 1: Characteristics of the patients included in the study

\begin{tabular}{|c|c|c|c|}
\hline \multicolumn{2}{|c|}{ Characteristics } & \multirow{2}{*}{$\begin{array}{l}\text { Number (\%) } \\
26(35.6 \%)\end{array}$} & \multirow{2}{*}{ Mean \pm SD } \\
\hline Gender & Male & & \\
\hline & Female & $47(64.4 \%)$ & ------- \\
\hline Age (years) & & ------- & $48.29 \pm 17.42$ \\
\hline \multirow[t]{4}{*}{ Co-morbidities } & Diabetes & $6(8.2 \%)$ & -------- \\
\hline & Hypertension & $19(26 \%)$ & ------- \\
\hline & $\begin{array}{l}\text { Coronary artery } \\
\text { disease }\end{array}$ & $6(8.2 \%)$ & ------- \\
\hline & COPD & $3(4.1 \%)$ & ------ \\
\hline Hospital stay & & ------- & $6.26 \pm 4.98$ \\
\hline Mortality & & $1(1.4 \%)$ & ------- \\
\hline
\end{tabular}

Sixty-five patients $(89 \%)$ had mild acute biliary pancreatitis, one (1.4\%) patient had moderately severe acute biliary pancreatitis, and $7(9.6 \%)$ patients had severe acute biliary pancreatitis. There was a development of complications in $7(9.6 \%)$ among severe acute biliary pancreatitis and none of the complications in mild or moderately severe acute biliary pancreatitis (figure 1). Among 7 complications, one had mortality secondary to acute pancreatic necrosis with acute respiratory distress syndrome (ARDS) and acute renal failure (ARF).

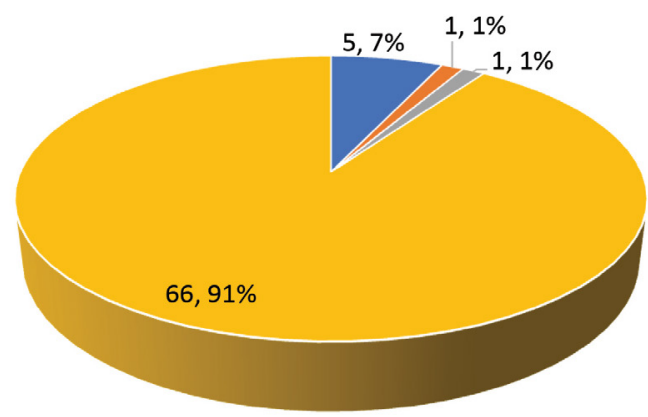

$$
\begin{aligned}
& \text { - Pleural effusion } \\
& \text { " Gl bleeding* } \\
& =\text { Acute pancreatic necrosis with ARDS and ARF* } \\
& =\text { No complications }
\end{aligned}
$$

* $G I=$ gastrointestinal bleeding, ARDS = acute respiratory distress syndrome, $A R F=$ acute renal failure.

Figure 1. Complications of acute biliary pancreatitis. 
The mean neutrophil to lymphocyte ratio (NLR) and mean platelet to lymphocyte ratio (PLR) were high in Group A (severe acute biliary pancreatitis) compared to Group B which included mild and moderately severe ABP, however, these differences were not statistically significant (Table 2).

Table 2: Neutrophil to lymphocyte ratio (NLR) and platelet to lymphocyte ratio (PLR) between the Groups.

\begin{tabular}{llll}
\hline \multicolumn{2}{c}{ Variables studied } & Mean \pm SD & p-value \\
\hline NLR & Group A & $6.09 \pm 3.71$ & 0.669 \\
& Group B & $5.58 \pm 3.36$ & \\
\hline \multirow{2}{*}{ PLR } & Group A & $176.61 \pm 139.01$ & 0.411 \\
& Group B & $131.63 \pm 63.04$ &
\end{tabular}

$p<0.05$ was considered as significant

\section{DISCUSSION}

Neutrophil to lymphocyte ratio (NLR) and platelet to lymphocyte ratio (PLR) has been studied as an inflammatory marker and correlated with systemic inflammatory reaction even in the absence of infection. ${ }^{7}$ Acute pancreatitis is an inflammatory disease and excessive inflammatory response leads to the destruction of the pancreas and organ failure. ${ }^{5}$ Therefore, this study was aimed to evaluate the correlation of NLR and PLR with the severity of acute pancreatitis due to gallstone.

In this study, NLR and PLR were high in severe acute biliary pancreatitis, however, these differences were not statistically significant ( $p=0.669$, and $p=0.411$ respectively). In contrast to our study, a study by Cho et al, which included acute pancreatitis due to gallstones and alcohol use, showed a correlation of NLR and PLR with severe acute biliary pancreatitis in subgroup analysis. But it fails to predict severe acute pancreatitis due to alcohol use. ${ }^{5}$

Kaplan Mustafa et al. found NLR and PLR as a predictor for severe acute pancreatitis and associated with the development of complications. ${ }^{8}$ Similarly, study by EKİN Nazim et al also found NLR and PLR as a predictor for severe acute pancreatitis. ${ }^{9}$
However, these studies have included patients with acute pancreatitis due to different etiology rather than acute biliary pancreatitis.

Huang Li et al analyzed the correlation of NLR with acute severe pancreatitis with different etiology (hyperlipidemia, alcohol, and gallstone) and they found NLR as a predictor for acute severe pancreatitis due to hyperlipidemia. NLR was not significantly correlated with the development of severe acute pancreatitis due to alcohol and gallstone. ${ }^{10}$ Similarly, a study done by İlhan Mehmetet et al showed a significant correlation of NLR with the severity of acute pancreatitis. However, PLR did not correlate with the severity of acute pancreatitis. ${ }^{11}$ Srikanth Reddy Challapalli studied correlation PLR with poor prognosis and outcome of acute pancreatitis. ${ }^{12}$

Studies to find out NLR and PLR as a predictor of severe acute pancreatitis included patients with different etiology of acute pancreatitis and there was heterogeneity in the definition of severe acute pancreatitis. These are the reason for the different findings of the different studies.

Though subgroup analysis of the study done by Cho Seung Kook $^{5}$ showed a significant correlation of NLR and PLR with the severity of acute biliary pancreatitis and severity was defined according to revised Atlanta classification as in our study, they did not mention cases associated with cholecystitis and acute cholangitis in which NLR and PLR may raise and may contribute in patient selection bias in the study. In our study, we excluded cases of acute pancreatitis associated with acute cholecystitis and acute cholangitis.

However, there are some limitations of our study that are the retrospective nature of the study, single centered study, and small sample size. Thus, a multicentered, well-designed prospective study is required to find out the use of NLR and PLR in predicting severe acute biliary pancreatitis.

\section{CONCLUSIONS}

Neutrophil to lymphocyte ratio (NLR) and platelet to lymphocyte ratio (PLR) could not predict severe acute biliary pancreatitis in our study.

\section{REFERENCES}

1. Husu HL, Leppäniemi AK, Lehtonen TM, Puolakkainen PA, Mentula PJ. Short-and long-term survival after severe acute pancreatitis: A retrospective 17 years' cohort study from a single center. J Crit Care. 2019;53:81-6. Crossref

2. Popa CC, Badiu DC, Rusu OC, Grigorean VT, Neagu SI, Strugaru CR. Mortality prognostic factors in acute pancreatitis. J med life. 2016;9(4):413. Website

3. Berger Z, Mancilla C, Tobar E, Morales MP, Baró M, Carrasco M, et al. Acute pancreatitis in Chile: a multicenter study on epidemiology, etiology and clinical outcome. Retrospective analysis of clinical files. Pancreatology. 2020;20(4):637-43. Crossref

4. Żorniak M, Beyer G, Mayerle J. Risk Stratification and Early Conservative Treatment of Acute Pancreatitis. Visc Med. 2019;35(2):82-9. $\underline{\text { Crossref }}$
5. Cho SK, Jung S, Lee KJ, Kim JW. Neutrophil to lymphocyte ratio and platelet to lymphocyte ratio can predict the severity of gallstone pancreatitis. BMC Gastroenterol. 2018;18(1):18. Crossref

6. Banks PA, Bollen TL, Dervenis C, Gooszen HG, Johnson CD, Sarr MG, et al. Classification of acute pancreatitis - 2012: revision of the Atlanta classification and definitions by international consensus. Gut. 2013 Jan;62(1): 102-11. Crossref

7. Fang T, Wang Y, Yin X, Zhai Z, Zhang Y, Yang Y, et al. Diagnostic sensitivity of NLR and PLR in early diagnosis of gastric cancer. J Immunol Res. vol. 2020, Article ID 9146042, 9 pages, 2020. Crossref

8. Kaplan M, Ates I, Oztas E, Yuksel M, Akpinar MY, Coskun O, Kayacetin E. A new marker to determine prognosis of acute pancreatitis: PLR and NLR combination. J MedBiochem. 2018 Jan;37(1):21. Website 
9. EKIN N, ARAÇ E, ARAÇ S. Can Hematological Parameters Predict the Severity of Acute Pancreatitis? Duzce Med J, 2020;22(2):79-83. Website

10. Huang L, Chen C, Yang L, Wan R, Hu G. Neutrophil-to-lymphocyte ratio can specifically predict the severity of hypertriglyceridemiainduced acute pancreatitis compared with white blood cell. J Clin Lab Anal. 2019;33(4):e22839. Crossref

11. İlhan M, İlhan G, Gök AF, Bademler S, VeritAtmaca F, Ertekin C. Evaluation of neutrophil-lymphocyte ratio, platelet-lymphocyte ratio and red blood cell distribution width-platelet ratio as early predictor of acute pancreatitis in pregnancy. J Matern Fetal Neonatal Med. 2016;29(9):1476-80. $\underline{\text { Crossref }}$

12. Reddy CS, Karimaddela K, Theja P, Prakash GV, Kumar PM. Role of platelet to lymphocyte ratio in assessing prognosis in acute pancreatitis. IntSurg. 2019;6(3):853-6. Crossref 